\title{
Construction and Analysis of Variable Wall Thickness Profile of Double Scroll Teeth of Scroll Compressor
}

\author{
Bin Peng ${ }^{1, *}$, Huixin Liu ${ }^{1}$, Pengcheng Zhang ${ }^{1}$,Yaohui Tao ${ }^{1}$ and Ting $\mathrm{Qi}^{1}$ \\ ${ }^{1}$ School of Mechanical and Electronical Engineering, Lanzhou University of Technology, Lanzhou 730050, P.R. China
}

\begin{abstract}
Based on the changing trend of the geometric parameters of the scroll profile of the involute of circle with variable radii, a new type of double scroll teeth profile of scroll compressor composed of involute of circle with variable radii is constructed. The construction process of the baseline is discussed, and the baseline equations are deduced. According to the principle of normal equidistance, the geometric model of variable wall thickness double scroll teeth is established, and the suction volume, tooth thickness, and scroll area of double scroll teeth are compared with those of the double scroll teeth composed of ordinary involute of circle. The results show that: compared with ordinary double scroll teeth with constant thickness, the scroll diameter when two scrolls mesh is reduced and the revolutions of orbiting scroll required in the working process are reduced and it is easy to select better scroll profile through adjusting the changing coefficient of the base circle radius $k$, which provides a reference for scroll machinery using environment-friendly working fluids.
\end{abstract}

\section{Introduction}

Scroll machinery is another new type of positive displacement compressor after reciprocating compressors, rotor compressors, and screw compressors. It is widely used in refrigeration systems and low-temperature waste heat power generation due to its simple structure, low noise, high efficiency, and light weight. In the system [1]. Especially at present, due to environmental problems and the ozone layer hole, environment-friendly working fluids have received extensive attention worldwide. However, refrigerants with low ODP and GWP values, such as $\mathrm{CO}_{2}$, require sufficient working pressure in refrigeration and air-conditioning systems [2-3], and the construction of the scroll profile is the key factor in determining it. In this paper, therefore, a kind of profile of variable wall thickness double scroll teeth profile is constructed to provide a reference for it.

At present, domestic and foreign scholars have done relatively little research on the double scroll teeth profile, most of which are involute of circle with the same wall thickness from start to the end. Wang Jun et al. used involute of circle with variable radii and algebraic spirals to construct double scroll teeth with gradually changing wall thickness that can achieve correct meshing, and gave profile equations [4]. Wang Jun et al. have been studied the working process of the compressor in detail [5]. Li Chao et al. analyzed the dynamic characteristics of the double scroll teeth compressor [6]. Li et al. studied the meshing characteristics and geometric theory of the involute of circle with variable radii, and obtained its conjugate meshing profile equation, and compared it with the involute of circle [7]. Peng Bin et al. proposed a combination curve of variable wall thickness scroll expander with involute of circle, high-order curve, and involute of circle, and deduced the expression of working chamber volume [8]. Wang Jun et al. established a general geometric model suitable for multiple scrolls with arbitrary teeth [9], Sun et al. used the finite element method to obtain the rough and finishing scroll entities and obtained the feed speed and excitation frequency fitting curve [10]. Liu proposed a combination of involute of circle, involute of circle with variable radii and involute of circle [11].

Although the above studies described the gradual changing wall thickness and variable wall thickness, most of the double scroll teeth adopt ordinary involute of circle to establish the model. This paper analyzes the changing trend of geometric parameters of the profile and constructs a double scroll teeth profile composed of involute of circle with variable radii.

\section{Geometrical model of involute of circle with variable radii}

\subsection{The geometric theory of profile}

The ordinary involute of circle has a constant base circle radius, on the contrary, base circle radius and polar diameter of this new type of profile are functions of the spread angle of the involute. The equations of base circle radius and polar diameter are as follows:

$$
R(\varphi)=R_{0}+k \varphi
$$

*Corresponding author: pengb2000@163.com 


$$
\rho(\varphi)=\int_{0}^{\varphi} R(\varphi) d \varphi=R_{0} \varphi+\frac{k}{2} \varphi^{2}
$$

\subsection{General equation of involute}

$$
\left\{\begin{array}{l}
X_{0}=\left(R_{0}+k \varphi\right) \cdot \cos (\varphi)+\left(R_{0} \varphi+\frac{k}{2} \varphi^{2}\right) \cdot \sin (\varphi) \\
Y_{0}=\left(R_{0}+k \varphi\right) \cdot \sin (\varphi)-\left(R_{0} \varphi+\frac{k}{2} \varphi^{2}\right) \cdot \cos (\varphi)
\end{array}\right.
$$

Where, $R_{0}$ is the initial base circle radius, $k$ is the changing coefficient of the base circle radius, $\varphi$ is the spread angle of involute.

The generated profiles with different value of $k$ are shown in Fig.1.

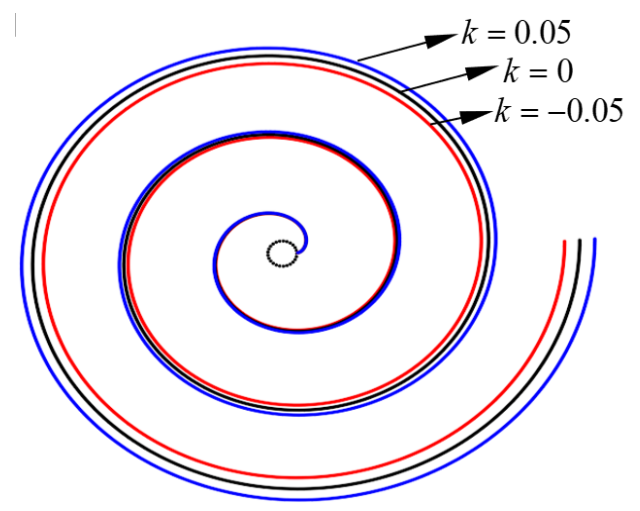

Fig 1. Schematic diagram of profiles with different value of $k$

\subsection{The changing trend of wall thickness and pitch with different value of $k$}
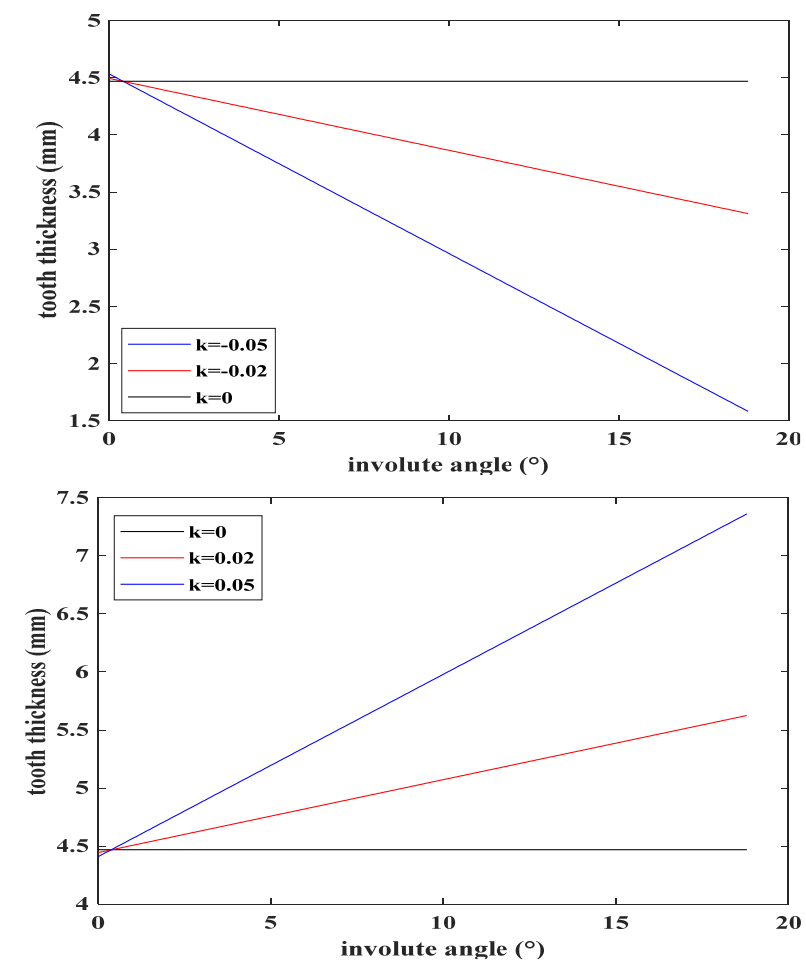
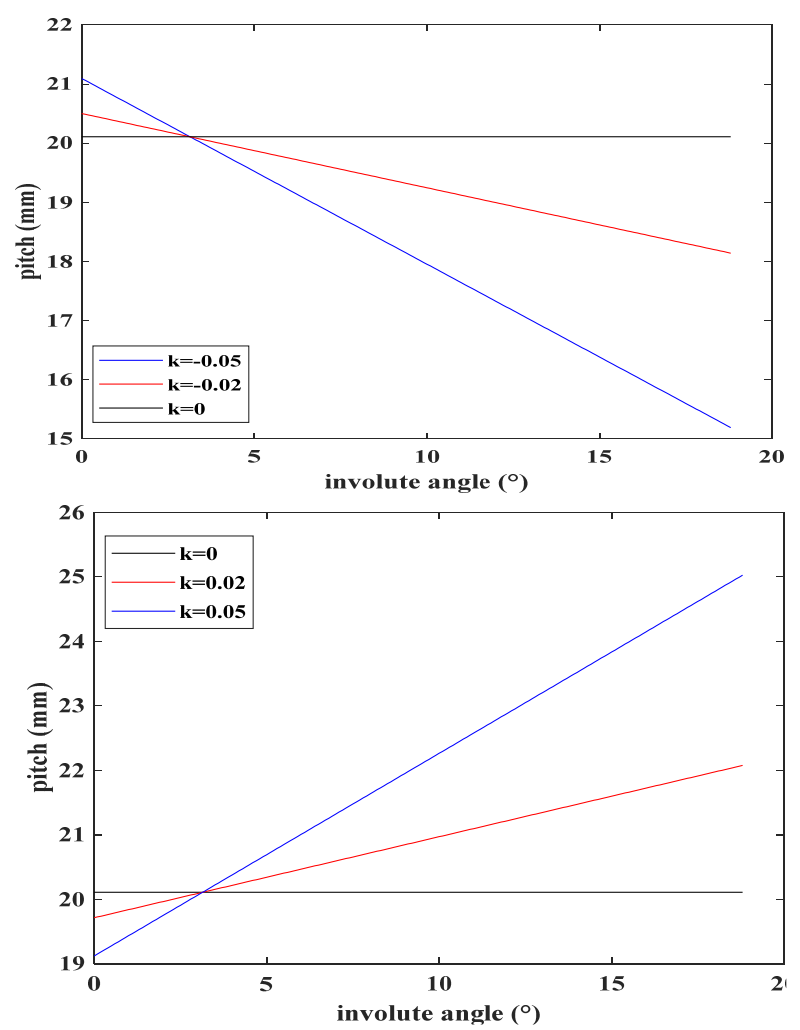

Fig 2. Schematic diagram of changing trends of wall thickness and pitch

It can be seen from the Fig.2. that when $k<0$, the tooth thickness and pitch decrease with the decrease of $k$; when $k>0$, the tooth thickness and pitch increase with the increase of $k$; the smaller the $k$, the smaller the corresponding overall wall thickness, the larger the $k$, the larger the corresponding overall wall thickness; and it can be clearly seen that when $k$ is near the involute spread angle $\varphi=0^{\circ}$, the tooth thickness increases with the decrease of $k$. From the above analysis, it is necessary to try to construct double scroll teeth constructed from this kind of profile.

\section{Conditions for the mutual substitution of the involutes of circle with variable radii with different initial base circle radii}

Select two involutes $S_{1}$ and $S_{2}$ with different initial base circle radii $R_{1}$ and $R_{2}$, the equations are as follows:

$$
\left.\begin{array}{c}
\left\{\begin{array}{l}
X_{1}=\left(R_{1}+k \varphi\right) \cdot \cos (\varphi)+\left(R_{1} \varphi+\frac{k}{2} \varphi^{2}\right) \cdot \sin (\varphi) \\
Y_{1}=\left(R_{1}+k \varphi\right) \cdot \sin (\varphi)-\left(R_{1} \varphi+\frac{k}{2} \varphi^{2}\right) \cdot \cos (\varphi)
\end{array}\right. \\
\varphi \in\left[\varphi_{1}, \varphi_{1}+2 n_{1} \pi\right]
\end{array}\right\}
$$

Where, ${ }^{\varphi, \varphi^{\prime}}$ is the spread angle of involute, $\varphi_{1}, \varphi_{2}$ is 
the arbitrary value of angle of $S_{1}$ and $S_{2}, n_{1}, n_{2}$ is the positive integer and $n_{1}>n_{2}$.

\subsection{The replacement process of baselines}

The processes of curve $S_{1}$ is replaced by $S_{2}$ are composed of rotation and translation. As shown in Fig.3. The initial positions of $S_{1}$ and $S_{2}$ are shown in (1); the position of curve $S_{2}$ after rotating $\varphi_{1}-\varphi_{2}$ around the origin of the coordinates is shown in the (2); and curve $S_{2}$ is marked as $S_{C D^{\prime}}$; the two points $A$ and $B$ coincide with the two points $C$ and $D$ through translation as shown in (3), then the curve $S_{2}$ is marked as $S_{C^{\prime \prime} D^{\prime \prime}}$, the replacement process ends.

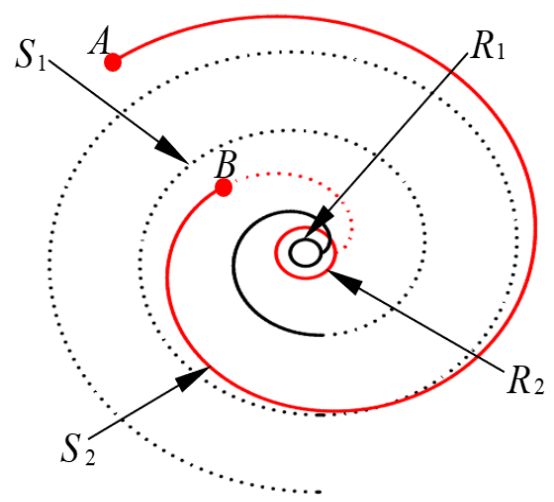

(1)

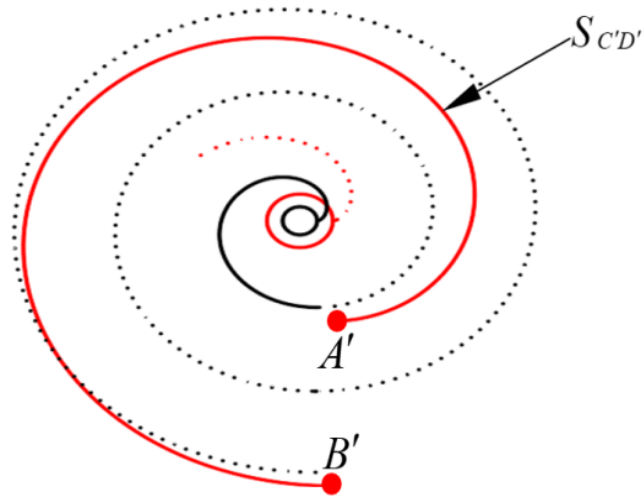

(2)

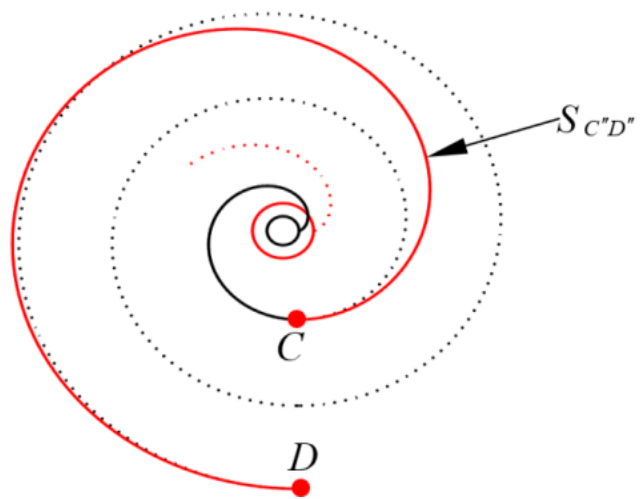

(3)

Fig 3. Schematic diagram of replacement process of $S_{1}$ and $S_{2}$

\section{2 replacement conditions}

Since the type of profile of involute of circle can maintain the same slope of the tangent at contact points during the rotation process, the replacement process can be completed only when the distance $L_{\mathrm{AB}}=L_{\mathrm{CD}}$ is satisfied. As shown in Fig.3., the curve segment $S_{\mathrm{CD}}$ on the baseline with the initial base circle radius of $R_{1}$ can be replaced by the curve segment $S_{\mathrm{AB}}$ on the baseline with the initial base circle radius of $R_{2}$. The following equation must be satisfied:

$$
\begin{aligned}
& \sqrt{\left[X_{2}\left(\varphi_{2}+2 n_{2} \pi\right)-X_{2}\left(\varphi_{2}\right)\right]^{2}+\left[Y_{2}\left(\varphi_{2}+2 n_{2} \pi\right)-Y_{2}\left(\varphi_{2}\right)\right]^{2}} \\
& =\sqrt{\left[X_{1}\left(\varphi_{1}+2 n_{1} \pi\right)-X_{1}\left(\varphi_{1}\right)\right]^{2}+\left[Y_{1}\left(\varphi_{1}+2 n_{1} \pi\right)-Y_{1}\left(\varphi_{1}\right)\right]^{2}}
\end{aligned}
$$

The above equation can be solved to get

$$
\frac{n_{1}^{2}}{n_{2}^{2}}=\frac{k^{2}+\left[R_{2}+k\left(\varphi_{2}+n_{2} \pi\right)\right]^{2}}{k^{2}+\left[R_{1}+k\left(\varphi_{1}+n_{1} \pi\right)\right]^{2}}
$$

\section{The establishment of variable wall thickness double scroll teeth}

\subsection{Basic theory of double scroll teeth}

For a compressor composed of a single scroll tooth, the two scrolls are installed with a phase difference of $180^{\circ}$. For double scroll teeth, two scroll teeth are symmetrically distributed around the origin on both orbiting and fixed scrolls, and these two scroll plates are installed with a phase difference of $90^{\circ}$. Therefore, the whole eight profiles can be calculated and drew through operations of isometry solid, rotation and mirroring in SOLIDWORKS when the baseline equation of orbiting or fixed scroll is given.

\subsection{The baseline equations of variable wall thickness double scroll teeth}

The first segment $S_{1}$ of baseline

$$
\left\{\begin{array}{c}
X=\left(R_{1}+k \varphi\right) \cos (\varphi)+\left(R_{1} \varphi+\frac{k}{2} \varphi^{2}\right) \sin (\varphi) \\
Y=\left(R_{1}+k \varphi\right) \sin (\varphi)-\left(R_{1} \varphi+\frac{k}{2} \varphi^{2}\right) \cos (\varphi) \\
\varphi \in\left[0, \varphi_{1}\right]
\end{array}\right.
$$

The second segment $S_{C^{\prime \prime} D^{\prime \prime}}$ of baseline

$$
\left\{\begin{array}{l}
X=\left[R_{2}+k(\varphi-\theta)\right] \cos (\varphi) \\
+\left[R_{2}(\varphi-\theta)+\frac{k}{2}(\varphi-\theta)^{2}\right] \sin (\varphi)-\left[X_{2}^{\prime}\left(\varphi_{1}\right)-X_{1}\left(\varphi_{1}\right)\right] \\
Y=\left[R_{2}+k(\varphi-\theta)\right] \sin (\varphi) \\
-\left[R_{2}(\varphi-\theta)+\frac{k}{2}(\varphi-\theta)^{2}\right] \cos (\varphi)-\left[Y_{2}^{\prime}\left(\varphi_{1}\right)-Y_{1}\left(\varphi_{1}\right)\right] \\
\quad\left[X_{2}^{\prime}\left(\varphi_{1}\right)-X_{1}\left(\varphi_{1}\right)\right]=\left[\left(R_{2}-R_{1}\right)-k \theta\right] \cos \left(\varphi_{1}\right) \\
+\left\{\left(R_{2}-R_{1}\right) \varphi_{1}-\left[R_{2}+\frac{k}{2}\left(2 \varphi_{1}-\theta\right)\right] \theta\right\} \sin \left(\varphi_{1}\right)
\end{array}\right.
$$




$$
\begin{gathered}
{\left[Y_{2}^{\prime}\left(\varphi_{1}\right)-Y_{1}\left(\varphi_{1}\right)\right]=\left[\left(R_{2}-R_{1}\right)-k \theta\right] \sin \left(\varphi_{1}\right)} \\
+\left\{\left(R_{2}-R_{1}\right) \varphi_{1}+\left[R_{2}+\frac{k}{2}\left(2 \varphi_{1}-\theta\right)\right] \theta\right\} \cos \left(\varphi_{1}\right) \\
\theta=\varphi_{1}-\varphi_{2} \\
\varphi \in\left[\varphi_{1}, \varphi_{1}+2_{n_{1}} \pi\right]
\end{gathered}
$$

The third segment $S_{1}$ of baseline

$$
\left\{\begin{array}{c}
X=\left(R_{1}+k \varphi\right) \cos (\varphi)+\left(R_{1} \varphi+\frac{k}{2} \varphi^{2}\right) \sin (\varphi) \\
Y=\left(R_{1}+k \varphi\right) \sin (\varphi)-\left(R_{1} \varphi+\frac{k}{2} \varphi^{2}\right) \cos (\varphi) \\
\varphi \in\left[\varphi_{1}+2 n_{1} \pi, \varphi_{e}\right]
\end{array}\right.
$$

Where, $\varphi_{e}$ is the ended involute angle.

The above baseline is rotated by $\pi$ around the origin of the coordinate to obtain another baseline segment of the scroll. The baseline of scroll is shown in Fig.4.

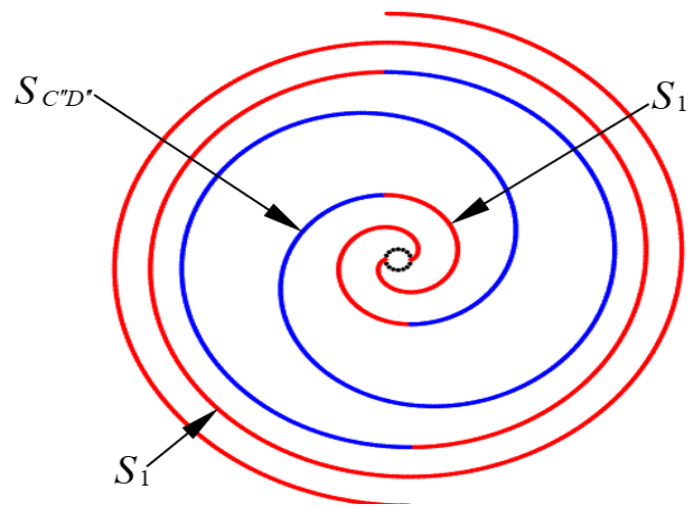

Fig 4. Schematic diagram of baseline of the scroll

\subsection{Comparison of geometric parameters of two double scroll teeth models}

According to the principle of normal equidistance, the initial base circle radii are chosen as $R=R_{1}=10 \mathrm{~mm}$, the number of turns $n_{1}, n_{2}$ are taken as 2 and $1, \varphi_{1}, \varphi_{2}$ are taken as $2 \pi$ and $1.2 \pi$, the ended involute angle and orbiting radius are taken as $\varphi_{e}=8 \pi$ and $R_{o r}=6 \mathrm{~mm}$ respectively, take $k=0, k=-0.05$ to generate the inner profiles of orbiting scroll and outer profiles of fixed scroll of ordinary double scroll teeth and the new type of double scroll teeth, which are installed with a phase difference of $0.5 \pi$ between the orbiting and fixed scrolls. As the research focus of this paper is on the profile itself, the correction of the teeth head has little influence on indexes such as suction volume, wall thickness and so on, the teeth head part is not modified. The two models are shown in Fig.5.:

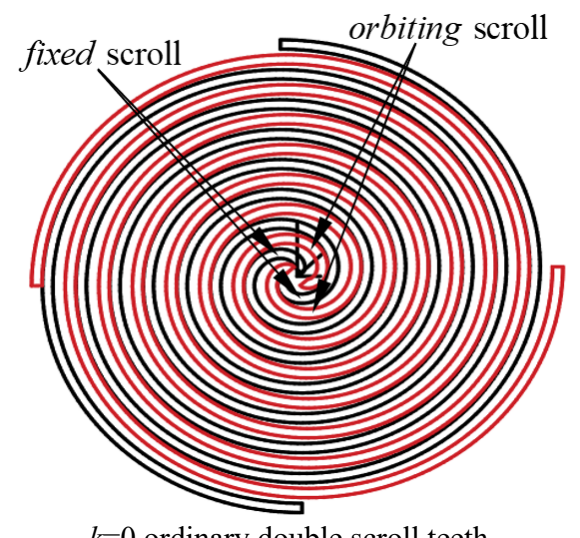

$k=0$ ordinary double scroll teeth

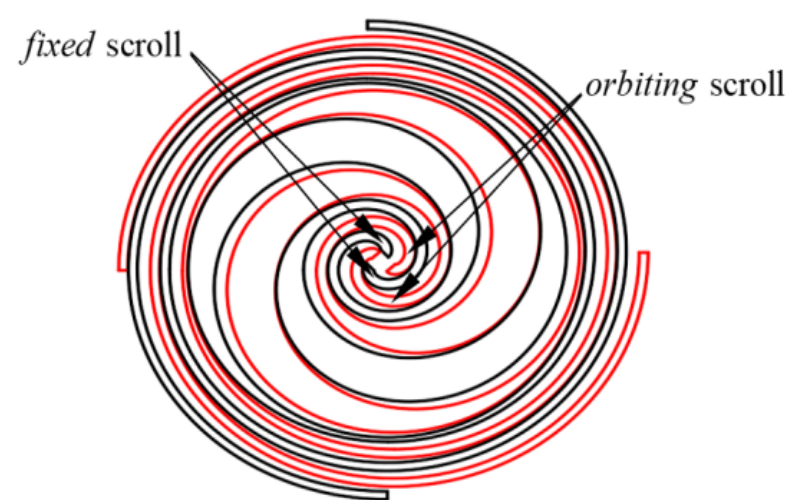

$k=-0.05$ variable wall thickness double scroll teeth

Fig 5. Comparison of two types of double scroll teeth

Because the double scroll teeth compressor has 4 suction chambers, the suction process is completed when the spindle rotates $0.5 \pi$. The volume of each suction chamber is the same. In order to simplify calculation, the projected area of one suction chamber is used for comparison. Through measurement, the projected areas of one suction chamber of $k=0$ and $k=-0.05$ are $7710.73 \mathrm{~mm}^{2}$ and $7253.92 \mathrm{~mm}^{2}$ respectively; the diameters of these two double scroll teeth during meshing are: $495.72 \mathrm{~mm}$ and $464.88 \mathrm{~mm}$; the areas of fixed scroll are $28023.32 \mathrm{~mm}^{2}$ and $28221.4 \mathrm{~mm}^{2}$; the minimum tooth thicknesses of teeth are $4.85 \mathrm{~mm}$ and $3.85 \mathrm{~mm}$, respectively. When the diameters of the two scroll teeth are the same value, the suction chamber projected area of $k=-0.05$ is $0.4 \%$ larger than that of the double scroll teeth of $k=0$, which can be almost ignored. However, with the gradual value decrease of $k$, the change will become greater and greater. Analyzing above data, it can be concluded that the plate diameter of the new type of double scroll teeth is reduced by $6.3 \%$; there is little difference in suction volume between them. However, the revolutions required for the orbiting scroll to complete the entire working process are reduced. It can be seen from Figure 4, the wall thickness of $k=-0.05$ in the middle compression chamber is significantly larger than that of $k=0$, which is more suitable for environmentfriendly working fluids that require high temperature and pressure operating conditions.

\section{Conclusions}

(1) The geometric model of involute of circle with 
variable radii is discussed, and the changing trend of its wall thickness and pitch is deduced. From which, variable wall thickness double scroll teeth composed of this profile is proposed.

(2) The conditions for the mutual substitution of profiles with different initial base circle radii are calculated, and the baseline equation of this new type profile of double scroll teeth is deduced, and the geometrical models of $k=0$ and $k=-0.05$ are established, through comparative analysis, it is concluded that the new profile can reduce the plate diameter and shorten the time that working fluid staying in the working chamber. According to actual working temperature and pressure, the appropriate scroll can be designed by adjusting the changing coefficient of the base circle radius $k$, which provides a certain reference for scroll machinery using environment-friendly working fluids.

\section{Acknowledgments}

This work was supported by the National Natural Science Foundation of China (Grant No.51675254, 51966009), the National Key Research and Development Program of China (Grant No. SQ2020YFF0420989), the Talent Innovation and Entrepreneurship Program of Lanzhou (Grant No.2020-RC-23), the Science and Technology Program of Gansu Province (Grant No.20YF8GA057).

\section{References}

1. B. Peng, Y. Sun. Investigation of mathematical modeling and experiment for variable thickness scroll compressor[J]. Journal of Mechanical Engineering 14, 185-191(2015).

2. Y.G. Liu et al. Optimum design of scroll profiles created from involute of circle with variable radii by using finite element analysis[J]. Mechanism and Machine Theory 55, 1-17(2012).

3. Y.G. Liu, C.H. Hung, Y.C, Chang. Study on involute of circle with variable radii in a scroll compressor[J]. Mechanism and Machine Theory 11,15201536(2010).

4. J. Wang, X.Q. Li. Construction of tapered thickness wrap profile for twin wrap scroll machinery[J]. Journal of China University of Petroleum(Edition of Nature Science) 03,128-132(2008).

5. J. Wang, X.Q. Li. The working process characteristic of multi-wraps scroll compressor[J]. Compressor Technology 02,17-19+22(2007).

6. C. L, et al. Analysis on dynamic characteristics of the twin-wraps scroll compressor[J]. Contemporary Chemical Industry 12, 2542-2545+2554(2014).

7. J. Wang, X.Q. Li. Investigation of profile of base circle involute with changing radius in scroll compressor[J]. Compressor Technology 04, 1$3+34(2011)$.

8. B. Peng, P.C. Zhang. Geometrical model and volume change of a variable cross section scroll expander[J].
Machine Design and Manufacture 06, 5-8(2019).

9. J. Wang, H. J, et al. Influence of wrap number on performance of multi-wraps scroll compressor[J]. Journal of China University of Petroleum(Edition of Nature Science) 03, 118-122(2009).

10. Y. J. Sun, T. Liu. Research on high speed machining complex profiles of scroll compressor[J]. Modern Manufacturing Engineering 07, 25-30+150(2017).

11. X. P. Liu, Q.Y. Li, et al. Design of a new type of combined profile scroll compressor[J]. Design and Research 03, 6-10(2019). 